\title{
Mathematical Model to Evaluate Behavior of Oil Spill Discharged into Tigris
}

River

$$
\text { بناء موديل رياضي لتحديد كيفية أنتشار البقع النفطية في نهر دجلة }
$$

Dr. Qays M. Ammouri ${ }^{*}$, Dr. Yousif J. Kazem ${ }^{* *}$, Nashwan Khudhair Mohammed ${ }^{* * *}$, Mays M. Abdul Kareem*, Hadeel A. Hussain*

*Iraqi Oil Ministry, PRDC, Baghdad

*** Ministry of oil, Baghdad

${ }^{* * *}$ Baghdad Oil Training Institute, Baghdad

الخلاصة

يتضمن البحث بناء موديل رياضي لتكهن سلوك تسرب بقعة نفطية إلى نهر دجلة ومصير تلك البقعة على طول مسار النهر. الموديل الرياضي أخذ بنظر الاعتبار معالجة مجموعة من العوامل مثل عامل انتشار البقعة النفطية على سطح الماء ومعدل التبخر ومعدل الاستحلاب للزيت في ماء النهر ، إضافة إلى انتشار البقعة على حافة النهر. ان حسابات الموديل اعتمدت بشكل اساسي على الته العو امل

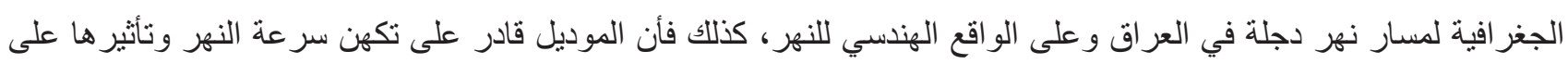

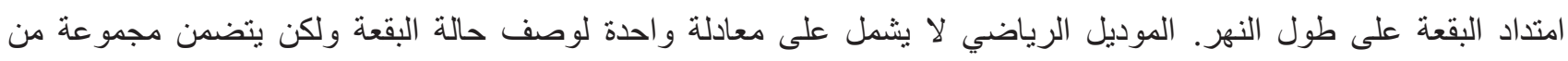

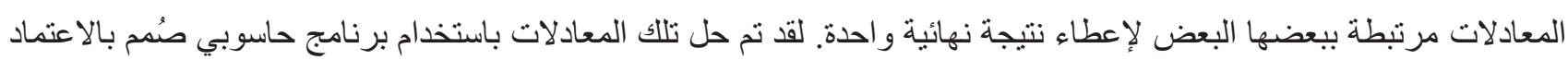
على لغه برمجية (فِجول بيسك) لتسهيل الحصول على النتائج من قِبل أي مستخدم.

\section{$\underline{\text { Abstract }}$}

This study included first attend in Iraq to built a mathematical model predicting the behavior oil spill discharged into Tigris river and the fate of spill along the river. The model included many factors such as spreading \& oil spill, evaporation; emulsion shoreline closes in river sedge. The calculations based on the geographical location of the spill \& geometry of the river section also, the model predicts the speed of Water River and its effect on the oil. Spill spreading along the river. The model did not describe the oil spill in one equation but, it divided to steps related together to give the single results. The model equation can be solved by computer software designed using visual basic program to facility getting result by any use.

\section{$\underline{\text { Introduction }}$}

Mathematical model of prediction the oil spill fate in river Tigris is achieved in this work, after that the model applied by a computer program designed by Visual Basic language ,to simplify the use of the mathematical model to any user [1]. 
River Tigris representing an important source of water for many governors in Iraq, it has long path from north to south, and it is very important to prevent and control any pollution accidents occurs in the river . oil spill one of most possible event in the river, this belong to the fact of many oil projects, like refineries storages and others are founded in different areas of Iraq close to the river and also many pipelines pass near it. In the past time there is no any record or archives for such events and there is no any scientific data about the oil spill happened in the river Tigris. Therefore, it is very important to build a mathematical model to control, predict and record all oil spill can occur in the future. The model cover the whole path of the river across Iraq lands from the entering point in the north Duhuk to the last point in the south Basra. Taking into account the weather effect, river water conditions, geometry of the river and oil spilled type and properties [2].

There are many oil spill studies and models. Almost all these models were developed for marine environment. For rivers, limited models were developed and the model can be applied for the specific river and this is the fact that each river differ than other rivers which required a limited oil spill mathematical model [3].

The oil spill in the river will take many directions, at the minute it starts, under the effect of the river conditions at the area of spill. First, advection under effect of river water velocity and wind speed take place, the velocity of oil and flow direction will determine the time required to reach specific place. Known this time will very important to treatment work. Second, the horizontal flow of the oil across the river and maximum diameter of the oil spill can give the idea about the area will be cover by the oil. Third, the effects of loss some hydrocarbons due to evaporation will be calculate by the mathematical model, which represent an important factor in the air pollution. The loss in the shorelines (river cliff) according to the nature and constituent the river cliff also the emulsion effect on oil properties with time to be evaluate mathematically [3].

Most of the above factors are calculated from experimental models used previously and derived from the basic of scientific resources. The final result of the work is a simple and clear computer application fed with oil spill data like location, date, oil spill amount, type of the oil, wind speed and weather conditions. All of this information are easy to collect and fed to the program [4].

Then the computer will provide results of spreading velocity and area, time to reach any area will be known, amount evaporated after time, the loss at river cliff, and changing in oil properties aftertime. Normally, the calculations is made for the first twelve hours due to the fact that the oil spill spreading will take its final limit within this time and the treatment work will be started at this period specially in the oil spill river it is more easier than oil spill in the sea [5]. 
At the end the program will keep a record of all details for this oil spill and this will be a basic archive to any future study or observation of oil spill pollution in the river Tigris [6].

\section{The Mathematical Model}

The mathematical model dialed with four points:

1. Evaluation of oil spill speed.

2. Evaluation of oil loss by evaporation.

3. Evaluation of oil deposed on river beaches.

4. Evaluation of oil residue.

In general, the state of oil spill transported on river water can be described by equation 1:

So, the quantity of oil transferred with river is calculated using equation 2:

$$
\begin{aligned}
& O_{\mathrm{S}}=\mathrm{O}_{\mathrm{A}}+\mathrm{O}_{\mathrm{V}}+\mathrm{O}_{\mathrm{SL}} \\
& \mathrm{O}_{\mathrm{A}}=\mathrm{O}_{\mathrm{S}}-\left(\mathrm{O}_{\mathrm{V}}+\mathrm{O}_{\mathrm{SL}}\right)
\end{aligned}
$$

\section{Transport and Diffusion}

When volume of oil discharged to river water, the first stage that described the behavior of the oil spill formed is the transport of oil spill with river current associated with diffusion of oil in river water.

The overall speed of oil spill transported over river water is yield of spill velocity with river current direction and molecular diffusion velocity of oil as shown in equation 3

$$
\mathrm{U}_{\mathrm{t}}=\mathrm{U}_{\mathrm{a}}+\mathrm{U}_{\mathrm{b}}
$$

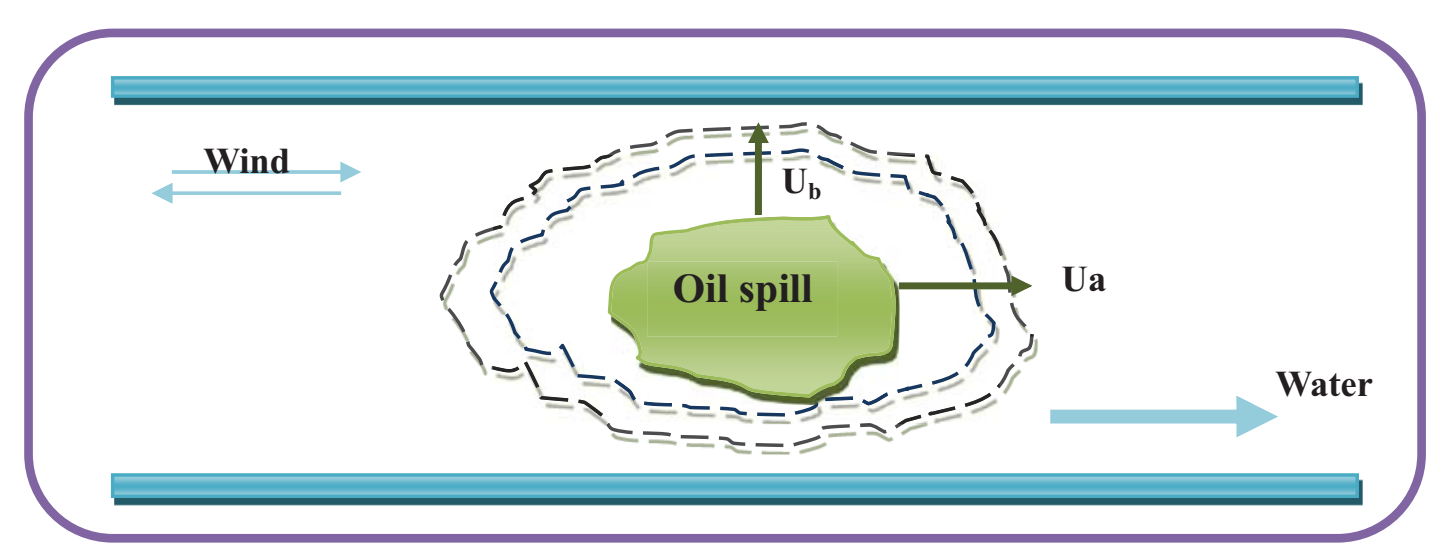

Fig. (1)

Spill velocity with river current direction depending on wind direction and illustrated by equations 4 and 5 : 


$$
\begin{aligned}
& \mathrm{U}_{\mathrm{a}}=\mathrm{K}_{\mathrm{S}} \mathrm{U}_{\mathrm{s}}+\mathrm{K}_{\mathrm{W}} \mathrm{U}_{\mathrm{W}} \cdot \\
& \mathrm{U}_{\mathrm{a}}=\mathrm{K}_{\mathrm{S}} \mathrm{U}_{\mathrm{s}}-\mathrm{K}_{\mathrm{W}} \mathrm{U}_{\mathrm{W}} \cdot
\end{aligned}
$$

The velocity of river is graduated from zero in river bottom to maximum value at river surface and can be represented mathematically depending on fluid flow phenomena as shown in Fig. (2).

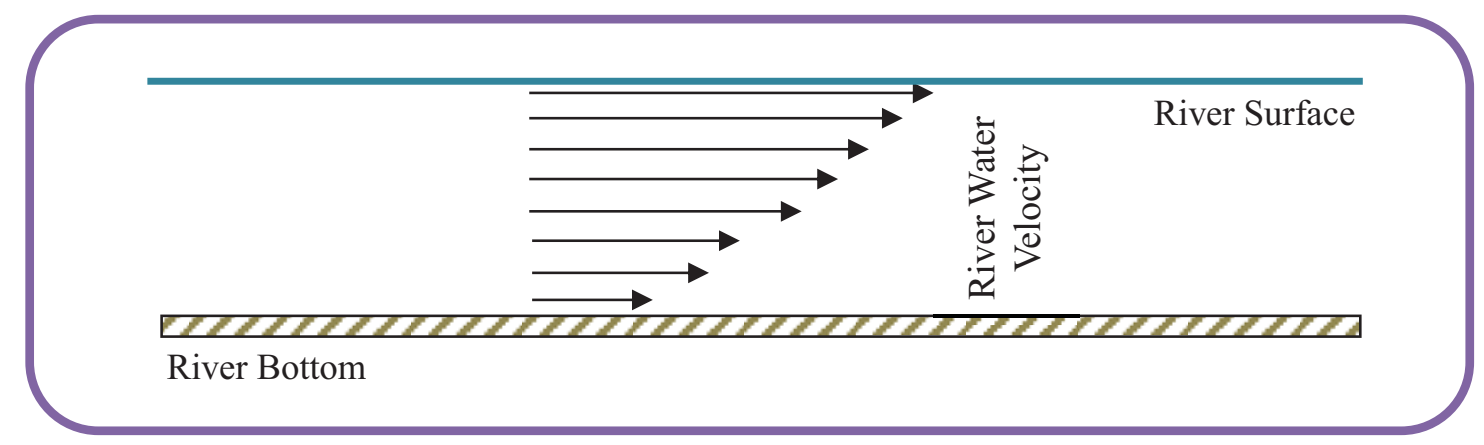

Fig. (2)

By taking slide with control volume $\Delta x \Delta y \Delta z$ shown in Fig. (3) and making momentum balance over it:

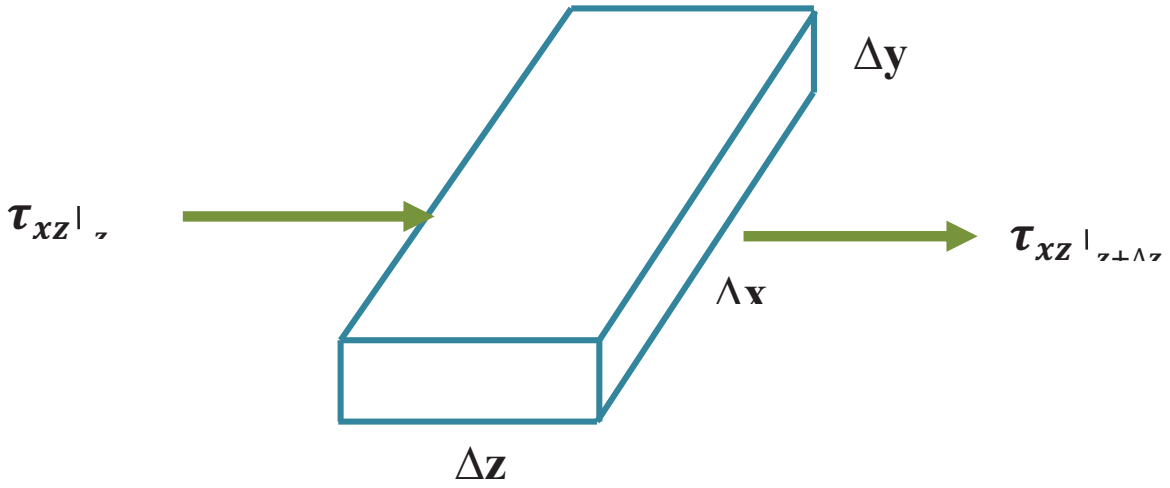

Fig. (3)

$$
\boldsymbol{\tau}_{x z_{z}} \cdot \Delta \mathrm{x} \Delta \mathrm{y}=\boldsymbol{\tau}_{\boldsymbol{x z}{ }_{\mathrm{z}+\Delta \mathrm{z}}} \cdot \Delta \mathrm{x} \Delta \mathrm{y}+\Delta \mathrm{x} \Delta \mathrm{y} \Delta \mathrm{z} \rho \mathrm{g} \theta \mathrm{z}
$$

At steady state:

Divided equation 6 on $\Delta \mathrm{x} \Delta \mathrm{y} \Delta \mathrm{z}$ :

Taking limit to equation 7 :

$$
\frac{\tau_{x z}}{\Delta z}=\frac{\tau_{x z} z+\Delta z}{\Delta z}+\rho g \theta z .
$$

According to Newton low: 


$$
\tau_{\mathrm{xz}}=-\mu \frac{d U_{Z}}{d z}
$$

Equation 8 becomes:

$$
\begin{aligned}
& \lim _{\Delta \mathrm{z} \rightarrow 0} \frac{\tau_{x z}{ }_{z}-\tau_{x z}{ }_{z+\Delta z}}{\Delta z}=\rho g \theta z \\
& \frac{\mathrm{d}}{\mathrm{dz}}\left(\tau_{\mathrm{xz}}\right)=\rho \mathrm{g} \theta \mathrm{z} \\
& \text { - } \frac{d}{d z}\left(\mu \frac{d U_{Z}}{d z}\right)=\rho g \theta \mathrm{z} \\
& \text { - } \frac{d}{d z}\left(\frac{d U_{z}}{d z}\right)=\frac{\rho \mathbf{g} \theta \mathbf{z}}{\mu} \\
& \frac{d^{2} \mathrm{U}_{\mathrm{z}}}{\mathrm{dz}^{2}}=-\frac{\rho g \theta z}{\mu} \text {. }
\end{aligned}
$$

Integration of equation 9 gives:

$$
U_{z}=-\frac{\rho g \theta}{2 \mu} \quad \mathrm{z}^{2}+\mathrm{C}_{\mathrm{z}}+\mathrm{D} .
$$

Equation 10 represents velocity distribution in dynamic water with z depth. By applying boundary conditions on equation 10 :

At $\mathrm{z}=0$ (River Bottom)

$$
\begin{gathered}
U_{z}=0 \\
0=0+0+\mathrm{D} \Rightarrow \mathrm{D}=0 \\
\mathrm{U}_{\mathrm{z}}=-\frac{\rho g \theta}{2 \mu} \mathrm{z}^{2}+\mathrm{C}_{\mathrm{z}}
\end{gathered}
$$

At $\mathrm{z}=\mathrm{h}$ (River Surface)

$$
\begin{gathered}
\frac{d U_{z}}{d z}=0 \\
\mathrm{Z}=\mathrm{h} \Longrightarrow \frac{d U_{z}}{d z}=0 \\
\frac{d u_{z}}{d_{z}}=-\frac{\rho g \theta}{2 \mu} z+C \\
\text { Then } \mathrm{C}=\frac{\rho g \theta h}{\mu} \\
\frac{d U_{z}}{d_{z}}=-\frac{\rho g \theta}{\mu} z+\frac{\rho g \theta}{\mu} h \\
\frac{d U_{z}}{d_{z}}=\frac{\rho g \theta}{\mu}(h-z) \ldots \ldots . . .
\end{gathered}
$$

Integration of equation 11:

$$
\begin{gathered}
\mathrm{U}_{\mathrm{z}}=\frac{\rho g \theta}{\mu}\left(z h-\frac{z}{2}^{2}\right) \\
\mathrm{U}_{\mathrm{z}}=\frac{\rho g \theta}{2 \mu} z(2 h-z)
\end{gathered}
$$




$$
\mathrm{U}_{\mathrm{s}}=\frac{\rho g \theta}{2 \mu} z(2 h-z)
$$

The evaluated velocity by equation 12 based on motion of river water in linear direction without any bending but in real state Tigris River move with bends inside Iraq as shown in Fig. (5) so, it's very important to correct the velocity.
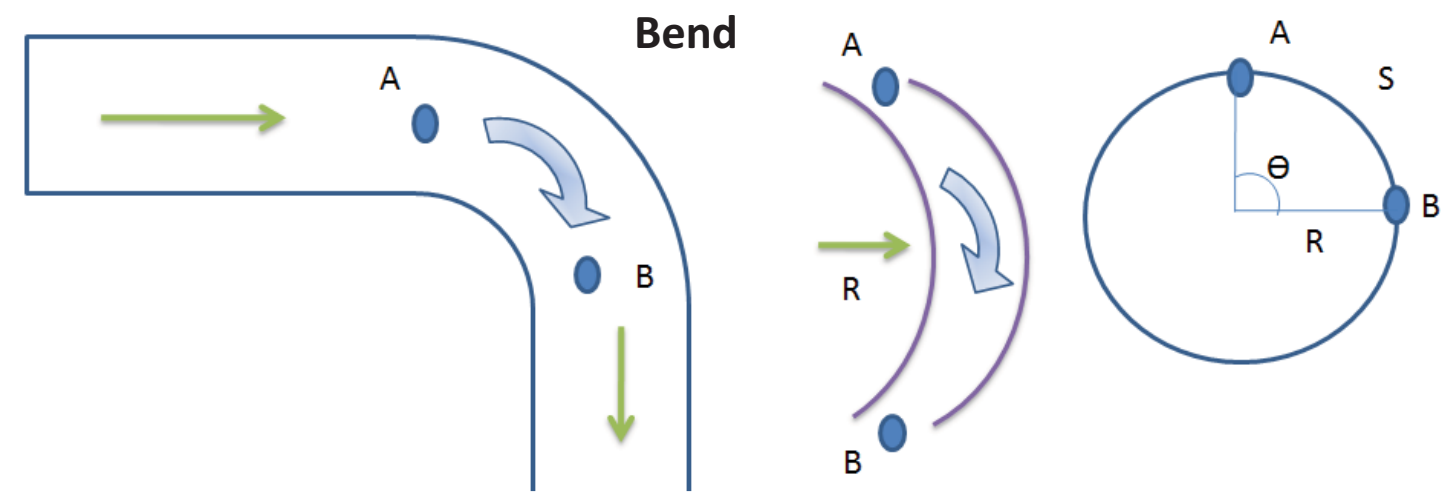

so:

Fig. (4)

$$
\begin{aligned}
& \mathrm{V}=\frac{S}{t} \\
& \omega=\frac{\theta^{\circ}}{t} . \\
& \omega=\frac{V}{R} .
\end{aligned}
$$

Where $i=1,2,3, \ldots$ etc

$$
\begin{gathered}
\omega_{\mathrm{i}}=\frac{V_{i}}{R_{i}} \\
\omega_{1}=\frac{U_{S}}{R_{1}} \\
\omega_{2}=\frac{\omega_{1}}{R_{2}} \\
\omega_{3}=\frac{\omega_{2}}{R_{3}} \\
\omega_{2}=\frac{\omega_{1}}{R_{2}}=\frac{\frac{U_{S}}{R_{1}}}{R_{2}}=\frac{U_{\mathrm{S}}}{R_{1} \times R_{2}} \\
\omega_{3}=\frac{\omega_{2}}{R_{3}}=\frac{\frac{U_{S}}{R_{1} \times R_{2}}}{R_{3}}=\frac{U_{\mathrm{S}}}{R_{1} \times R_{2} \times R_{3}}
\end{gathered}
$$

When

$$
\begin{aligned}
\mathrm{R}=\mathrm{R}_{1} & =\mathrm{R}_{2}=\mathrm{R}_{3} \\
\omega_{\mathrm{i}} & =\frac{U_{S}}{R_{1} \times R_{2} \times R_{3} \times \cdots \cdots R_{i}}
\end{aligned}
$$




$$
\begin{gathered}
\mathrm{U}^{\prime}=\frac{\omega_{1+\omega_{2}+\omega_{3}+\ldots \omega_{i}}}{i} \\
\mathbf{U}^{\prime}=\frac{\frac{U_{S}}{R_{2}}+\frac{U_{S}}{R_{1} \times R_{2}}+\frac{U_{S}}{R_{1} \times R_{2} \times R_{3}}+\ldots \omega_{i}}{i} \\
\mathbf{U}^{\prime}=\frac{U_{S} \sum \frac{1}{R^{i}}}{i} \ldots \ldots \ldots(18)
\end{gathered}
$$

For example:

$$
\mathbf{U}^{\prime}=\frac{U_{S}\left(\frac{1}{R_{1}}+\frac{1}{\left(R_{1} \times R_{2}\right)}+\frac{1}{\left(R_{1} \times R_{2} \times R_{3}\right)}+\frac{1}{\left(R_{1} \times R_{2} \times R_{3} \times R_{4}\right)}\right)}{4}
$$

At other hand, the molecular diffusion velocity of oil can be calculated using equation 19:

$$
\mathrm{V}_{\mathrm{b}}=\sqrt{4 D_{T} / \delta_{t}}
$$

The diffusivity coefficient is calculated by equation 20 :

$$
\mathrm{D}_{\mathrm{T}}=0.0372 \mathrm{n}_{\mathrm{b}} \mathrm{U}_{\mathrm{s}} \mathrm{g}^{1 / 2} \mathrm{~h}^{5 / 6}
$$

To evaluate molecular diffusion velocity it is important to evaluate random function as shown in Fig. (5):

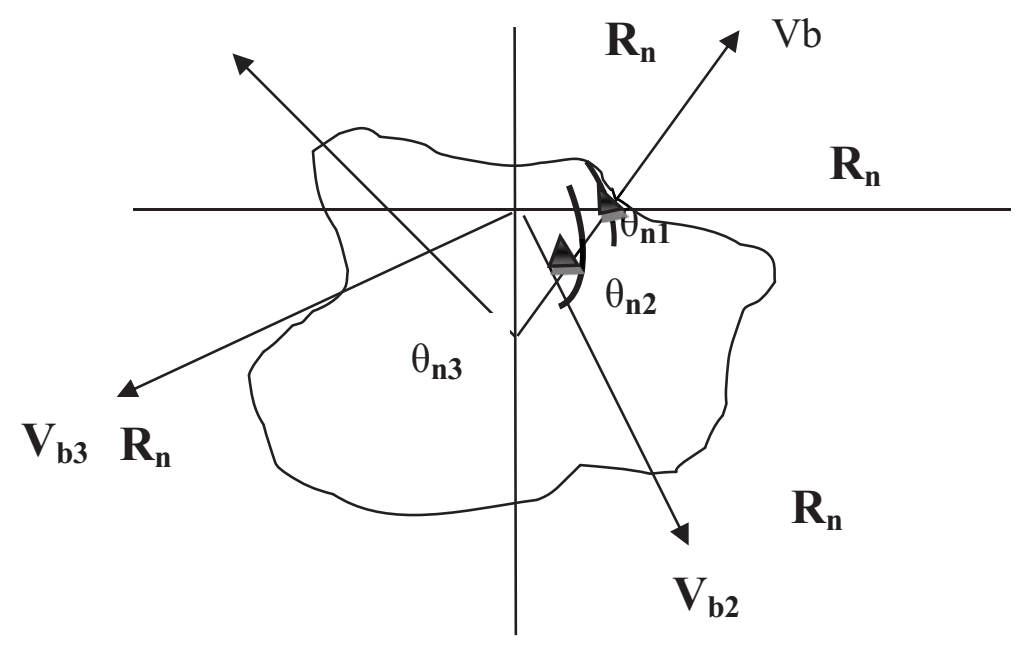

Fig. (5)

The final value of molecular diffusion velocity can be evaluated using equation 21 :

$$
\mathrm{U}_{\mathrm{b}}=\mathrm{R}_{\mathrm{n}} \mathrm{V}_{\mathrm{b}} \mathrm{e}^{\mathrm{i} \Theta \mathrm{n}}
$$

\section{Evaporation}

When oil spill move on river water, the light compounds of oil may evaporate, so the rate of evaporation is convicted with mass transfer low:

$$
\begin{array}{r}
\text { Input }=\text { Output }+ \text { Accumulation } \\
\left.N_{A}\right|_{X} \cdot \mathrm{A}=\left.N_{A}\right|_{X+\Delta X} \cdot \mathrm{A}+\mathrm{A} \cdot \Delta \mathrm{x} \cdot \frac{d C_{A}}{d t} \ldots \ldots .(22)
\end{array}
$$




$$
\begin{aligned}
& \left(N_{A \mid X}-\left.N_{A}\right|_{X+\Delta X}\right) \cdot \mathrm{A}=\mathrm{A} \cdot \Delta \mathrm{x} \cdot \frac{d C_{A}}{d t} \\
& -\frac{N_{A \mid X}-N_{A} \mid X+\Delta X}{\Delta \mathrm{x}}=\frac{d C_{A}}{d t} \\
& \lim _{\Delta \mathrm{x} \rightarrow 0} \frac{N_{A \mid X}-N_{A} \mid X+\Delta X}{\Delta \mathrm{x}}=\frac{d C_{A}}{d t} \\
& \quad-\frac{d}{d X}\left(\mathrm{~N}_{\mathrm{A}}\right)=\frac{d C_{A}}{d t}
\end{aligned}
$$

Substitution of Fick's low in equation 23:

$$
\begin{gathered}
\mathrm{N}_{\mathrm{A}}=-\mathrm{D} \frac{d C_{A}}{d X} \\
-\frac{\mathrm{d}}{\mathrm{dX}}\left(-\mathrm{D} \frac{\mathrm{dC}_{\mathrm{A}}}{\mathrm{dX}}\right)=\frac{\mathrm{dC}_{\mathrm{A}}}{\mathrm{dt}} \ldots \ldots .
\end{gathered}
$$

Applying equation 24 for component i:

$$
\frac{\mathrm{dC}}{\mathrm{dt}}=\mathrm{D}_{\mathrm{i}} \frac{d^{2} C_{i}}{d X^{2}}
$$

Solution of equation 25 is:

$$
\mathrm{c}_{\mathrm{i}}=c_{i^{\circ}} \operatorname{erfc}\left(\frac{X}{2 \sqrt{D_{i} t}}\right)
$$

The mole rate of evaporation is:

$$
\begin{gathered}
\mathrm{N}_{\mathrm{i}}=\mathrm{k}_{\mathrm{m}}\left(c_{i^{\circ}}-\mathrm{c}_{\mathrm{i}}\right) \\
\mathrm{K}_{\mathrm{m}}=0.0292 \mathrm{U}_{\mathrm{w}}^{0.78} \mathrm{~h}_{\mathrm{s}}^{-0.11} \mathrm{~S}_{\mathrm{c}}{ }^{-0.67} \\
\mathrm{~S}_{\mathrm{c}}=\frac{\mu_{i}}{\rho_{i D_{i}}} \ldots \ldots \ldots \ldots \ldots \ldots . . .
\end{gathered}
$$

According to equations 27 and 28 the rate of evaporation in mole/sec units can be calculated as follows:

$$
\mathrm{N}_{\mathrm{i}}=\mathrm{k}_{\mathrm{m}} C_{i^{\circ}}\left(1-\operatorname{erfc}\left(\frac{X}{2 \sqrt{D_{i} t}}\right)\right.
$$

While the rate of evaporation in $\mathrm{m}^{3} / \mathrm{sec}$ :

$$
\mathrm{V}_{\mathrm{i}}=\mathrm{K}_{\mathrm{m}} \frac{\rho^{\circ}{ }_{i}}{R T}\left(1-\operatorname{erfc} \quad\left(\frac{X}{2 \sqrt{D_{i} t}}\right)\right)
$$

\section{$\underline{\text { Emulsion }}$}

The motion of river water creates emulsion of water drops in oil, this emulsion system as shown in Figs.(6) and (7). 


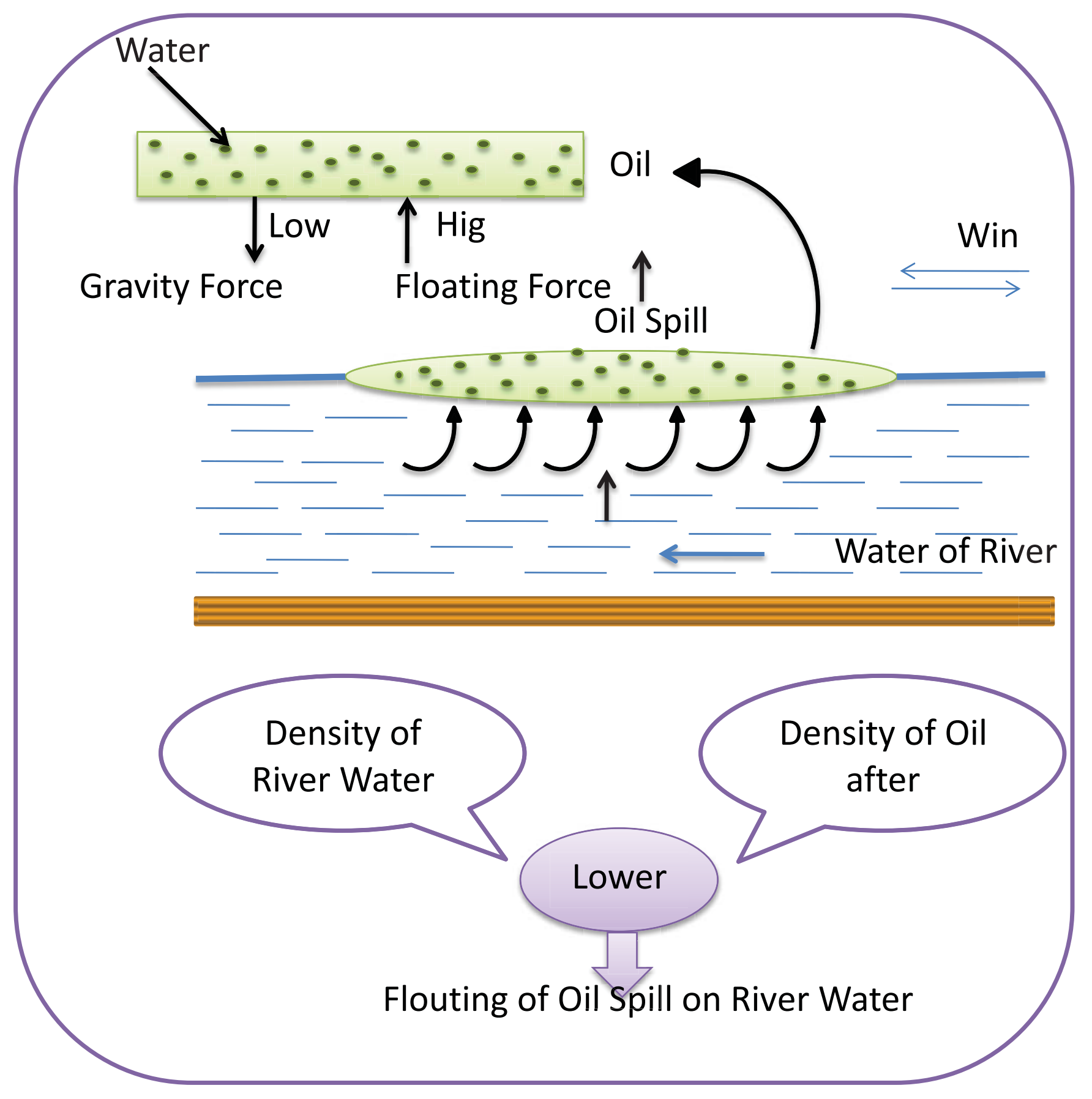

Fig. (6) 


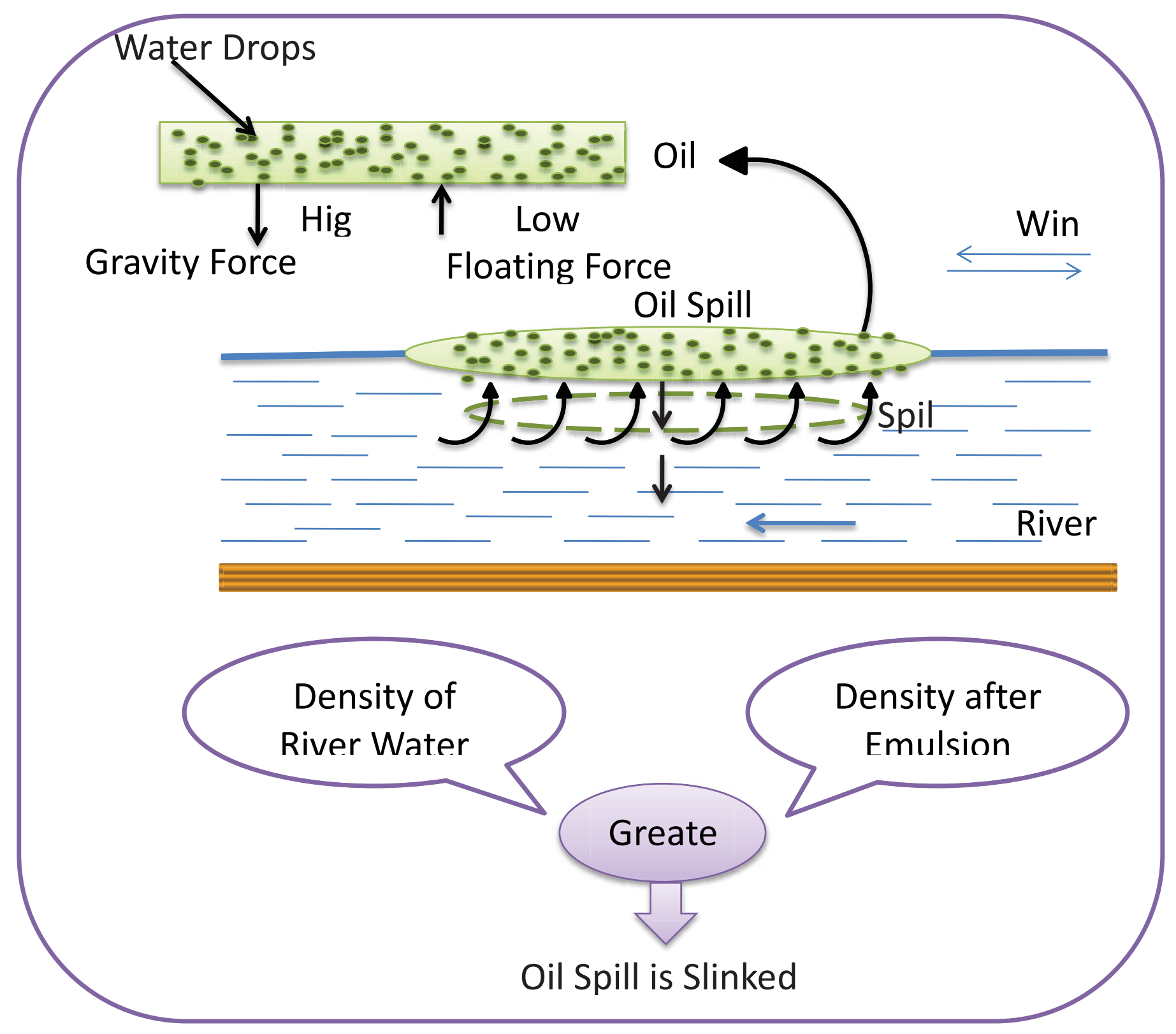

Fig. (7)

The water cut emulsified in oil phase must evaluated in order to know the fate of the spill:

$$
\frac{d v_{e}}{d t}=\mathrm{k}_{\mathrm{em}}\left(\mathrm{U}_{\mathrm{w}}+1\right)^{2}\left(1-\frac{v_{e}}{C_{3}}\right) .
$$

$\mathrm{k}_{\mathrm{em}}$ is constant can be calculated from experiments.

$$
\frac{d v_{e}}{d t}=\mathrm{k}_{\mathrm{em}}\left(\mathrm{U}_{\mathrm{w}}+1\right)^{2}-\frac{\mathrm{k}_{\mathrm{em}}\left(\mathrm{U}_{\mathrm{W}}+1\right)^{2} v_{e}}{\mathrm{C}_{3}}
$$

Multiplying equation 32 by $\mathrm{C}_{3}$ :

$$
\mathrm{C}_{3} \cdot \frac{d v_{e}}{d t}=\mathrm{k}_{\mathrm{em}}\left(\mathrm{U}_{\mathrm{w}}+1\right)^{2} \cdot \mathrm{C}_{3}-\mathrm{k}_{\mathrm{em}}\left(\mathrm{U}_{\mathrm{w}}+1\right)^{2} v_{e}
$$




$$
\begin{gathered}
\mathrm{C}_{3} \cdot \frac{d v_{e}}{d t}=\mathrm{k}_{\mathrm{em}}\left(\mathrm{U}_{\mathrm{w}}+1\right)^{2}\left[\mathrm{C}_{3}-v_{e}\right] \\
\mathrm{C}_{3} \cdot \frac{\mathrm{d} v_{\mathrm{e}}}{\mathrm{C}_{3}-v_{e}}=\mathrm{k}_{\mathrm{em}}\left(\mathrm{U}_{\mathrm{w}}+1\right)^{2} \mathrm{dt} \\
\frac{\mathrm{d} v_{\mathrm{e}}}{\mathrm{C}_{3}-v_{e}}=\frac{\mathrm{k}_{\mathrm{em}}}{\mathrm{C}_{3}}\left(\mathrm{U}_{\mathrm{w}}+1\right)^{2} \mathrm{dt} \ldots \ldots \ldots .(33)
\end{gathered}
$$

Taking integration of equation 33 :

$$
\begin{gathered}
\int_{0}^{v_{e}} \frac{\mathrm{d} v_{\mathrm{e}}}{\mathrm{C}_{3}-v_{e}}=\frac{\mathrm{k}_{\mathrm{em}}}{\mathrm{C}_{3}}\left(\mathrm{U}_{\mathrm{w}}+1\right)^{2} \int_{0}^{t} d t \\
\ln \frac{\mathrm{C}_{3}-v_{e}}{\mathrm{C}_{3}}=-\frac{\mathrm{k}_{\mathrm{em}}}{\mathrm{C}_{3}}\left(\mathrm{U}_{\mathrm{w}}+1\right)^{2} \mathrm{t} \\
\frac{\mathrm{C}_{3}-v_{e}}{\mathrm{C}_{3}}=\operatorname{EXP}\left(-\frac{\mathrm{k}_{\mathrm{em}}}{\mathrm{C}_{3}}\left(\mathrm{U}_{\mathrm{w}}+1\right)^{2} \mathrm{t}\right) \\
1-\frac{v_{e}}{\mathrm{C}_{3}}=\operatorname{EXP}\left(-\frac{k_{e m}(\mathrm{Uw}+1)^{2}}{\mathrm{C}_{3}}\right) t \\
\frac{v_{e}}{\mathrm{C}_{3}}=1-\operatorname{EXP}\left(-\frac{\mathrm{k}_{\mathrm{em}}}{\mathrm{C}_{3}}\left(\mathrm{U}_{\mathrm{w}}+1\right)^{2} \cdot \mathrm{t}\right) \\
v_{e}=\mathrm{C}_{3}\left[1-\operatorname{EXP}\left(-\frac{\mathrm{k}_{\mathrm{em}}}{\mathrm{C}_{3}}\left(\mathrm{U}_{\mathrm{w}}+1\right)^{2} \cdot \mathrm{t}\right)\right] \ldots . .
\end{gathered}
$$

Evaluation of water cut emulsified in oil phase leads to evaluation the density of oil after emulsion:

$$
\begin{aligned}
& \rho_{\mathrm{e}}=v_{e} \rho_{\mathrm{w}}+\left(1-v_{e}\right)\left(\rho_{\mathrm{c}}+\mathrm{C}_{3} \mathrm{~F}_{\mathrm{e}}\right) \\
& \rho_{\mathrm{e}}>\rho_{\mathrm{c}} \quad \Longrightarrow \text { Settling } \\
& \rho_{\mathrm{e}}<\rho_{\mathrm{c}} \quad \Longrightarrow \text { Floating }
\end{aligned}
$$

\section{Oil Deposited on River Beaches}

When oil discharged to water river, the oil spill is expanded in two dimensions as show in Fig. (8). The rate of oil deposited on the beaches of river can be evaluated as follows:

$$
\frac{\mathrm{d} v_{\mathrm{SL}}}{\mathrm{dt}^{\circ}}=\mathrm{k} v_{S L}
$$

$\mathrm{k}$ is constant depending on saturation half time of beach and type of beach, so:

$$
\begin{gathered}
\frac{v_{S L}}{\mathrm{dt}^{\circ}}=\mathrm{kdt} \mathrm{t}^{\circ} \\
\int_{v_{S L 1}}^{v_{S L 2}} \frac{d v_{S L}}{v_{S L}}=\mathrm{k} \int_{t^{\circ} 1}^{t^{\circ}} \mathrm{dt}^{\circ} \\
\ln \frac{v_{S L 2}}{v_{S L 1}}=\mathrm{k}\left(\mathrm{t}_{2}-\mathrm{t}_{1}\right)=\mathrm{k} \Delta \mathrm{t}^{\circ} \\
\frac{v_{S L 2}}{v_{S L 1}}=e^{k \Delta t^{\circ}} \ldots \ldots .(37)
\end{gathered}
$$



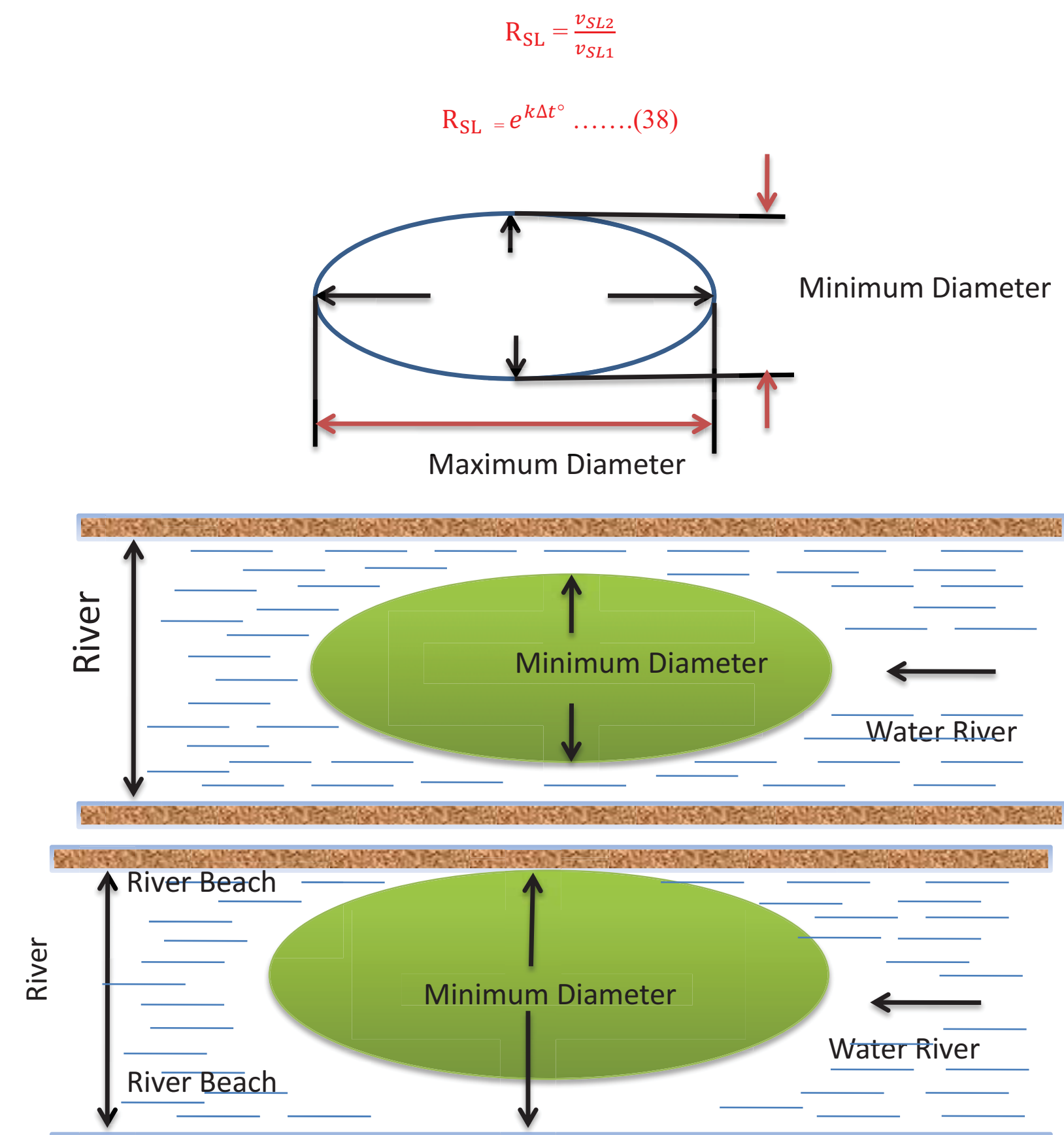

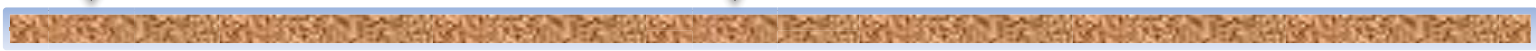

Minimum Diameter $>=$ River Beach

$\mathrm{R}_{\mathrm{SL}}=$ Value
Minimum Diameter $<$ River Beach $\mathrm{R}_{\mathrm{SL}}=$ Zero

Fig. (8) 


\section{Solution of Mathematical Model}

The derived equations of the model are complex, so the solution of this set of equations needs using of many boundary conditions. According to this reason computer package was designed in order to facile the solution of model equations and predict fate of oil spill discharged to Tigris river.

The computer package showing in Figs.(9) and (10) can be evaluating behavior and fate of oil spill according to block diagram showing in Fig.(11) that includes steps of operation this program.

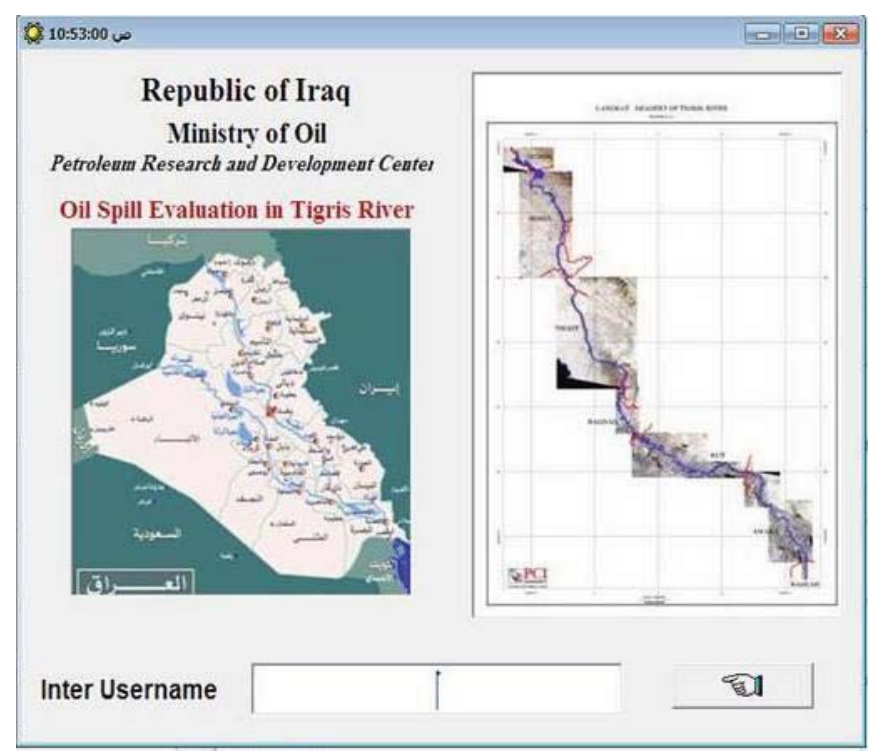

Fig. (9)

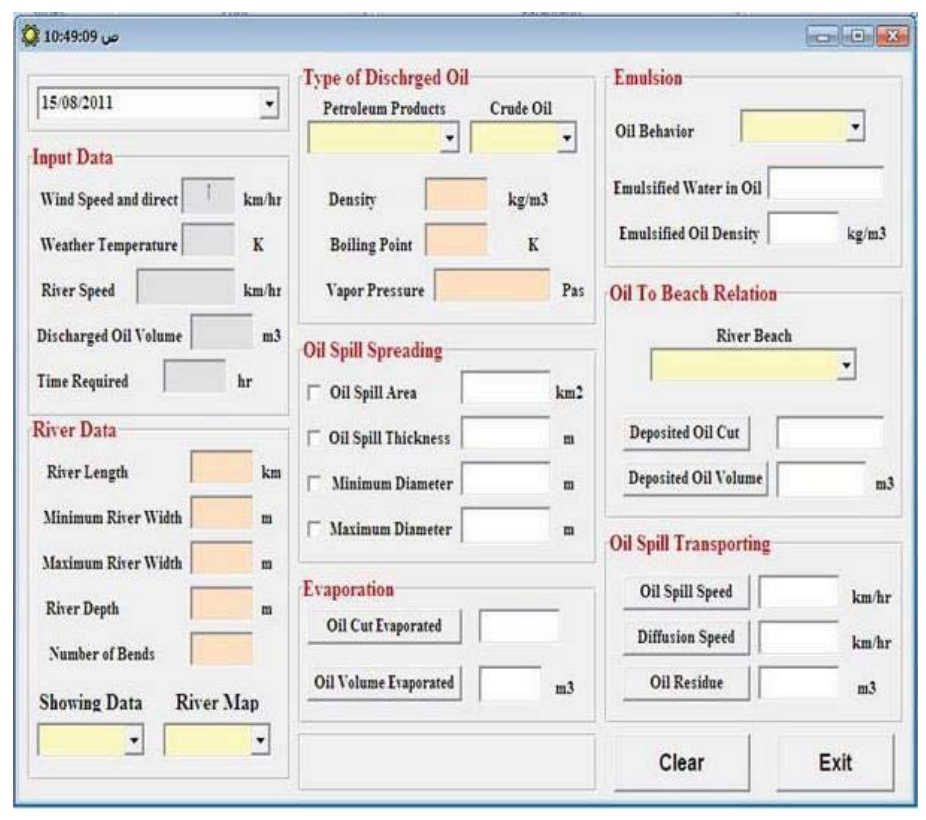

Fig. (10) 


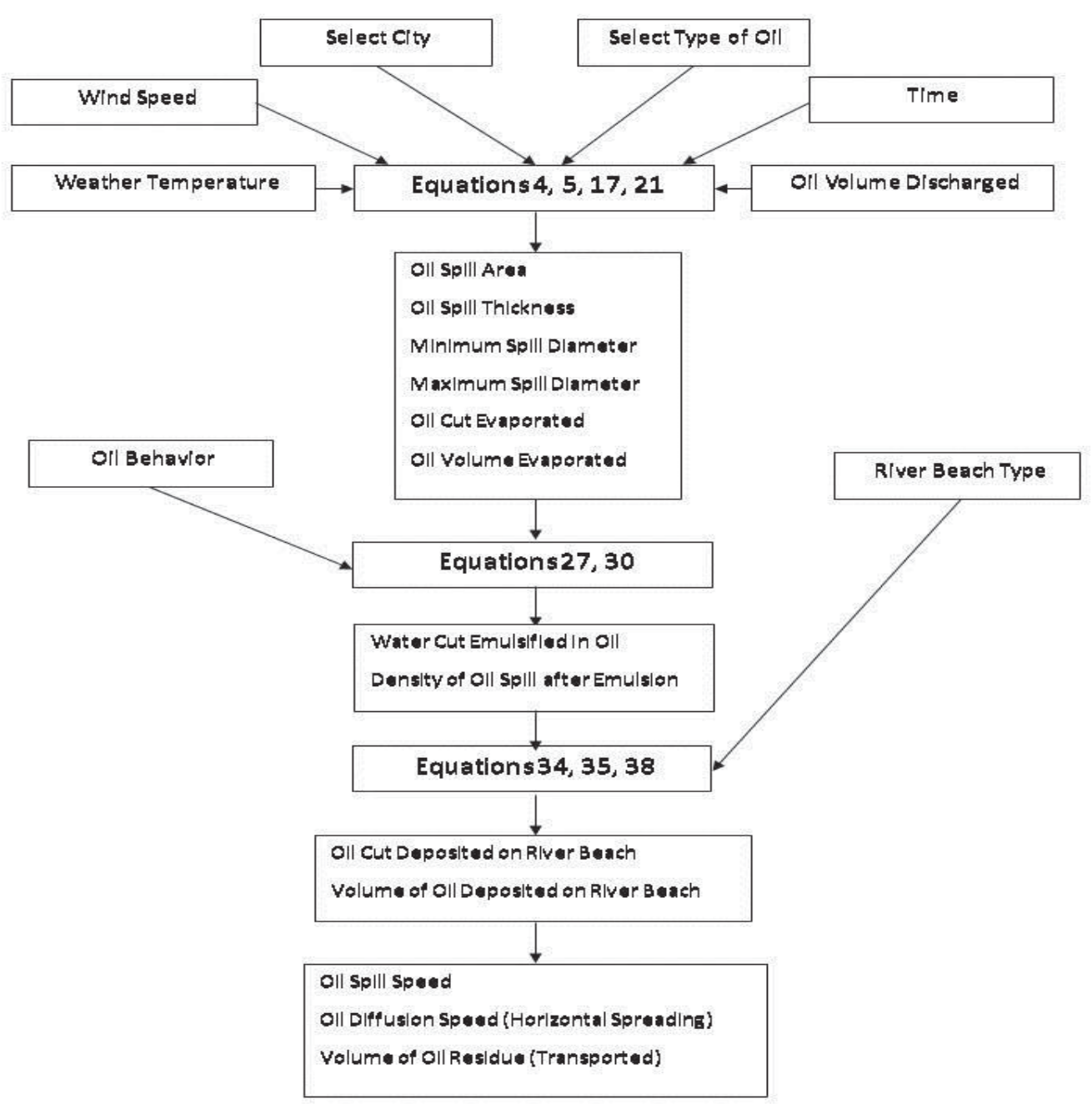

Fig. (11) 


\section{Nomenclatures}

\begin{tabular}{|c|c|c|}
\hline Symbol & Definition & Unit \\
\hline $\mathrm{O}_{\mathrm{s}}$ & Quantity of Oil Discharged & \\
\hline $\mathrm{O}_{\mathrm{v}}$ & Quantity of Oil Evaporated & \\
\hline $\mathrm{O}_{\mathrm{sL}}$ & Quantity of Oil Deposited on River Beaches & \\
\hline $\mathrm{O}_{\mathrm{A}}$ & Oil Transported with Water River Currents & \\
\hline $\mathrm{U}_{\mathrm{t}}$ & Total Transport Velocity of Oil Spill & $\mathrm{m} / \mathrm{Sec}$ \\
\hline $\mathrm{U}_{\mathrm{a}}$ & Speed of Oil Spill & $\mathrm{m} / \mathrm{Sec}$ \\
\hline $\mathrm{U}_{\mathrm{b}}$ & Diffusion Velocity of Oil Horizontally & $\mathrm{m} / \mathrm{Sec}$ \\
\hline $\mathrm{K}_{\mathrm{s}}$ & Constant & \\
\hline $\mathrm{K}_{\mathrm{w}}$ & Constant & \\
\hline $\mathrm{U}_{\mathrm{s}}$ & Speed of Water at Top River Surface & $\mathrm{m} / \mathrm{Sec}$ \\
\hline $\mathrm{U}_{\mathrm{w}}$ & Speed of Wind at $10 \mathrm{~m}$ over Sea Level & $\mathrm{m} / \mathrm{Sec}$ \\
\hline$\tau_{\mathrm{xk} z}$ & Input Moment at Distance z & $\mathrm{N} / \mathrm{Sec}$ \\
\hline$\tau_{\mathrm{xz}} \mathrm{z}^{+}+\Delta \mathrm{z}$ & Output Moment at Distance $\mathrm{z}+\Delta \mathrm{z}$ & $\mathrm{N} / \mathrm{Sec}$ \\
\hline $\mathrm{h}$ & River Depth & $\mathrm{m}$ \\
\hline$\rho$ & Density of Water & $\mathrm{Kg} / \mathrm{m}^{3}$ \\
\hline$\mu$ & Viscosity of Water & Pas.Sec \\
\hline $\mathrm{Z}$ & Distance toward Top Surface of River & $\mathrm{m}$ \\
\hline$\Theta$ & Angle of River Slop $\cong$ zero & \\
\hline $\mathrm{g}$ & Gravity Acceleration & $\mathrm{m} / \mathrm{Sec}^{2}$ \\
\hline $\mathrm{S}$ & Radical Distance of River & $\mathrm{m}$ \\
\hline $\mathrm{R}$ & Radius of River Bending & $\mathrm{m}$ \\
\hline$\theta^{\circ}$ & Bending Angle & \\
\hline $\mathrm{V}$ & Linear Velocity of River Water & $\mathrm{m} / \mathrm{Sec}$ \\
\hline $\mathrm{t}$ & Required Time & $\mathrm{Sec}$ \\
\hline $\mathrm{W}$ & Radiant Velocity of River & $\mathrm{m} / \mathrm{Sec}$ \\
\hline ú & Average Flow rate of RIVER & $\mathrm{m} / \mathrm{Sec}$ \\
\hline $\mathrm{i}$ & Number of Bends & \\
\hline $\mathrm{V}_{\mathrm{b}}$ & Diffusion Velocity & $\mathrm{m} / \mathrm{Sec}$ \\
\hline $\mathrm{D}_{\mathrm{T}}$ & Diffusivity & $\mathrm{m}^{2} / \mathrm{Sec}$ \\
\hline $\boldsymbol{\delta}_{\mathrm{t}}$ & Time Interval & \\
\hline $\mathrm{n}_{\mathrm{b}}$ & Roughness Factor of River Bottom & \\
\hline $\mathrm{U}_{\mathrm{s}}$ & Speed of River Water at Top Surface & $\mathrm{m} / \mathrm{Sec}$ \\
\hline $\mathrm{R}_{\mathrm{n}}$ & Random Number & \\
\hline$\Theta_{\mathrm{n}}$ & Random Angle & \\
\hline Di & Diffusivity Factor of Component i & $\mathrm{m}^{2} / \mathrm{Sec}$ \\
\hline $\mathrm{Z}$ & Distance of Evaporation through Air & $\mathrm{m}$ \\
\hline
\end{tabular}




\begin{tabular}{|c|c|c|}
\hline $\mathrm{C}_{\mathrm{i}}$ & Concentration of component $\mathrm{i}$ in Spill at Time $\mathrm{t}$ & $\mathrm{mol} / \mathrm{m}^{3}$ \\
\hline $\mathrm{C}_{\mathrm{i}^{\circ}}$ & Concentration of component $\mathrm{i}$ in Spill at Time zero & $\mathrm{mol} / \mathrm{m}^{3}$ \\
\hline erfc & Error Function Mathematical Formula & \\
\hline $\mathrm{X}$ & Thickness Distance of Oil Spill & $\mathrm{m}$ \\
\hline $\mathrm{N}_{\mathrm{i}}$ & Molar Diffusion Rate of Component i & $\mathrm{mol} / \mathrm{Sec}$ \\
\hline $\mathrm{K}_{\mathrm{m}}$ & Mass Transfer Coefficient & $\mathrm{m} / \mathrm{Sec}$ \\
\hline hs & Final Thickness of Oil Spill & $\mathrm{m}$ \\
\hline $\mathrm{S}_{\mathrm{c}}$ & Schmidth Number & \\
\hline$\mu_{i}$ & Viscosity of Oil & Pas.Sec \\
\hline$\rho_{i}$ & Density of Oil & $\mathrm{Kg} / \mathrm{m}^{3}$ \\
\hline $\mathrm{V}_{\mathrm{i}}$ & Rate of Evaporation & $\mathrm{m}^{3} / \mathrm{Sec}$ \\
\hline$\rho_{i}^{o}$ & Vapor Pressure of Compound i & Pascal \\
\hline $\mathrm{R}$ & Gas Constant & $\mathrm{J} / \mathrm{mol} . \mathrm{K}$ \\
\hline $\mathrm{T}$ & Weather Temperature & $\mathrm{K}$ \\
\hline $\mathrm{V}_{\mathrm{e}}$ & Cut of Emulsified Water in Oil & \\
\hline$\gamma_{\mathrm{o} / \mathrm{w}}$ & Surface Tension of Oil in Water & Dyen $/ \mathrm{cm}^{2}$ \\
\hline$\gamma_{\mathrm{w} / \mathrm{o}}$ & Surface Tension of Water in Oil & Dyen $/ \mathrm{cm}^{2}$ \\
\hline $\mathrm{C}_{3}$ & Constant & \\
\hline $\mathrm{k}_{\mathrm{em}}$ & Constant & \\
\hline$\rho_{\mathrm{e}}$ & Density of Oil Spill Before Emulsion & $\mathrm{Kg} / \mathrm{m}^{3}$ \\
\hline$\rho_{\mathrm{c}}$ & Density of Oil Spill after Emulsion & $\mathrm{Kg} / \mathrm{m}^{3}$ \\
\hline $\mathrm{F}_{\mathrm{e}}$ & Ratio of Oil Evaporated from Spill & $\mathrm{mol} / \mathrm{Sec}$ \\
\hline $\mathrm{V}_{\mathrm{SL} 1}$ & Volume of Oil Deposited on River Beach at Time $\mathrm{t} 1$ & $\mathrm{~m}^{3}$ \\
\hline $\mathrm{V}_{\mathrm{SL} 2}$ & Volume of Oil Deposited on River Beach at Time $\mathrm{t} 2$ & $\mathrm{~m}^{3}$ \\
\hline $\mathrm{t}^{\circ}$ & Time & $\mathrm{hr}$ \\
\hline $\mathrm{K}$ & Constant Depending on Half - Time of River Beach & \\
\hline
\end{tabular}




\section{References}

1. Stolzenbach K.D, O.S. Madsen, Adams, AM. Pollack and C.K. Cooper (1977) A review and evaluation of basic techniques for predicting the behavior of surface oil slicks. Department of civil engineering. Massachusetts institute of technology, Cambridge, Massachusetts, report no. 22 .

2. Huang, j.C., and F.C Monastero (1982) review of the state - of - the - art of oil spill simulation models .final report submitted to the American petroleum institute, June .Spaulding (1988).

3. Spaulding, M.L. (1988)A State -of the -art review of oil spill trajectory and fate modeling .oil and chemical pollution , 4: 39-55.

4. Shen, H.T., and P.D yapa (1988) oil slick transport in rivers . journal of hydraulic engineering, ASCE ,114(5):529-543.

5. Cronk, J.C., W.J. Mitsch and R.M. sykes , (1990), "efective modeling of a major inland oil spill on the Ohio river", Elsevier Science publishers B.V., Ecological Modeling , 51 , 161 192.

6. Nagheeby, M. and M. Kolahdoozan,(2010),'Numerical modeling of two fluid flow and oil slick transport in estuarine water". International journal of environment science and technology, Vol.7, No.4, pp. 771-784. 